\title{
Le timbre dans le répertoire de la valiha, cithare tubulaire de Madagascar
}

Jobonina Razafindrakoto

\section{(2) OpenEdition}

\section{Journals}

Édition électronique

URL : http://journals.openedition.org/ethnomusicologie/847

ISSN : 2235-7688

Éditeur

ADEM - Ateliers d'ethnomusicologie

Édition imprimée

Date de publication : 1 janvier 1999

Pagination : 123-142

ISBN : 978-2-8257-0680-0

ISSN : $1662-372 X$

\section{Référence électronique}

Jobonina Razafindrakoto, «Le timbre dans le répertoire de la valiha, cithare tubulaire de Madagascar », Cahiers d'ethnomusicologie [En ligne], 12 | 1999, mis en ligne le 08 janvier 2012, consulté le 30 avril 2019. URL : http://journals.openedition.org/ethnomusicologie/847

Ce document a été généré automatiquement le 30 avril 2019

Tous droits réservés 


\title{
Le timbre dans le répertoire de la valiha, cithare tubulaire de Madagascar
}

\author{
Jobonina Razafindrakoto
}

1 La cithare tubulaire de Madagascar est un instrument de musique fort ancien qui aurait été introduit sur l'île par des populations originaires du Sud-Est asiatique au cours du premier millénaire après J.-C. L'instrument se compose d'un tuyau de bambou sur lequel sont tendues parallèlement des cordes habituellement pincées avec les doigts (fig. 1). Appelée valiha, vadiha, betoroky ou encore marovany selon les régions, la cithare tubulaire est liée aux modes de vie des divers groupes ethniques qui peuplent la Grande Ile (Domenichini 1983 : 705-706). Sa pratique est particulièrement vivace en Imerina, région des Hautes Terres centrales de Madagascar : en témoignent les quelque 34 dénominations vernaculaires et d'emprunt que j'ai répertoriées pour qualifier la valiha chez les Merina (habitants de l'Imerina) ${ }^{1}$.

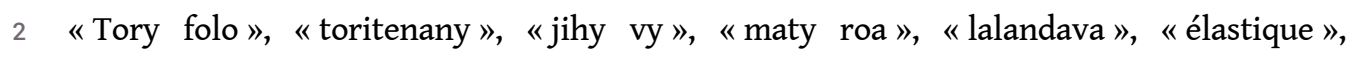
«électrique ", « sympathique ", « chromatique » ... forment un ensemble de qualificatifs juxtaposés au mot valiha que j'ai recueillis au cours de missions effectuées entre 1990 et 1995. Si rendre compte d'une réalité sonore consiste à noter les éléments musicalement significatifs pour les musiciens d'une culture donnée, ces 34 appellations constituaient de prime abord un corpus terminologique susceptible de renseigner sur la pratique de la valiha.

Une analyse organologique poussée montre que ces termes désignent des matériaux de fabrication, des dispositifs particuliers, des modalités d'accords ou encore des formes singulières: dix-sept qualificatifs se rapportent aux cordes de l'instrument, dix-sept autres à son corps de résonance (Razafindrakoto 1997 : 100-174). C'est ainsi que mon approche du répertoire a commencé: observer l'instrument de musique, source génératrice de sons. Cette diversité de facture pose aujourd'hui des problèmes d'ordre esthétique, en particulier celui du renouvellement de la matière sonore dans un répertoire de musique traditionnelle. 
Mon propos n'est pas d'avancer des solutions immédiates sur la notation mais, à la lueur de mon expérience de terrain et de mes résultats d'analyses, de poser une problématique sur un aspect particulier du son : que décrire, que noter à propos du timbre? Pour tenter de cerner une question aussi vaste, il faudrait faire une première approche analytique du timbre en cherchant sa nature dans celle de ses éléments constitutifs.

\section{Les timbres de la valiha}

Les musicologues distinguent quatre constituants élémentaires de la matière sonore : la hauteur, la durée, l'intensité et le timbre. Or, si les trois premiers sont traditionnellement représentés par les signes de la notation solfégique, il n'en va pas aussi simplement pour le quatrième. Aussi, afin de mieux cerner les problèmes relatifs à sa notation, est-il nécessaire de savoir de quoi le timbre est constitué d'une part, et s'il est immuable ou variable d'autre part. Pour cela, l'acoustique musicale va nous donner un accès spécifique à cet élément sonore. L'analyse du timbre instrumental revient donc à saisir au mieux les ressources acoustiques de l'instrument : d'abord, en caractérisant la "palette sonore " c'est-à-dire le matériau de base constitué par l'ensemble des sonorités dont le musicien dispose (Schaeffer 1966 et Chion 1983); ensuite, en analysant l'utilisation musicale de ce matériau. Cette recherche pluridisciplinaire que j'ai menée sur la valiha a requis le concours du physicien acousticien, de l'artisan-musicien et de l'ethnomusicologue.

Fig. 1 : La valiha

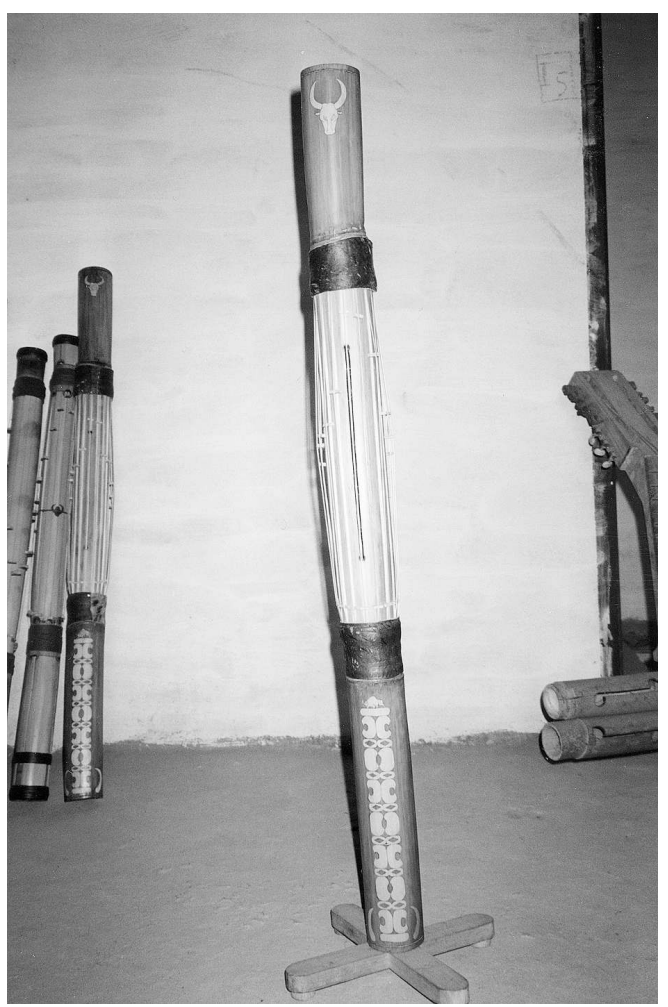

La comparaison entre divers enregistrements de valiha surprend par d'énormes différences de sonorités (Duvelle 1963 et Domenichini 1985). En effet, l'organologie instrumentale distingue deux grands types : la valiha ancienne dont les cordes végétales sont incisées et soulevées du corps même de l'instrument, et la valiha moderne dont les 
cordes métalliques sont rapportées au corps de résonance (Sachs 1938). Mon propos n'est pas d'en faire ici une étude organologique détaillée, mais de me demander quel est l'impact de ces transformations sur le répertoire traditionnel de l'instrument.

7 Mon objectif est donc de caractériser ces deux types d'instruments, leurs sonorités et la musique qu'ils produisent, d'abord sur des critères objectivement mesurables, ensuite sur les critères propres à la société merina afin de comprendre les choix de facture des artisans-musiciens.

\section{Comparaisons entre la valiha à cordes végétales et la valiha à cordes métalliques}

Le timbre est une donnée multidimentionnelle que l'on peut définir comme suit :

«[...] c'est essentiellement un attribut relevant simultanément de toutes les dimensions sonores: fréquence, amplitude, durée. Ce que nous appelons communément le timbre comporte en fait deux aspects [...]. Le premier se réfère à l'identification de la source sonore (causalité) et le second aux changements de qualités que le signal peut subir selon l'espace et le temps. Nous employons le terme «sonorité » pour l'aspect qualitatif, réservant celui de "timbre » pour l'aspect d'identification » (Castellengo $1994: 206)$.

9 Cette définition justifie l'étude de groupes et de familles instrumentales d'une part, et d'« êtres sonores » à l'intérieur de chaque famille d'autre part.

10 L'étude comparative des deux types de valiha, à cordes végétales (valiha toritenany) et à cordes métalliques (valiha jihy vy), a débuté avec la participation de musiciens malgaches au Laboratoire d'Acoustique Musicale (LAM) de l'Université Paris VI, dont un des axes de recherche concerne l'étude du fonctionnement des instruments de musique.

\section{L'analyse sonagraphique}

11 Le recueil des données en chambre sourde, puis l'analyse au sonagraphe audionumérique 5500 ont permis d'obtenir une description fine des paramètres qui régissent le timbre et sa perception auditive, à savoir : l'évolution temporelle, le contenu spectral et l'intensité des composantes.

12 A la lecture d'un sonagramme, la durée est représentée en secondes sur l'abscisse: transitoire d'attaque et transitoire d'extinction sont les phases dynamiques du son qui encadrent une tenue plus ou moins longue qui est la phase stable du son. Les fréquences exprimées en Hertz sont reportées en ordonnée: l'équidistance des composantes spectrales traduit un rapport simple entre les harmoniques, alors qu'une nonéquidistance des raies traduit un rapport complexe entre les partiels. Le son est perçu avec un maximum d'intensité lorsqu'il atteint la zone sensible de l'oreille située entre 2000 et 4000 Hertz. Enfin, l'intensité sonore mesurée en décibels est représentée par l'épaisseur des composantes spectrales ou des zones plus denses appelées formants.

13 Les sonagrammes des deux types de valiha font apparaître des différences spectrales très nettes. Le spectre harmonique de la valiha à cordes végétales (fig. 2) se caractérise par une attaque franche qui s'apparente à un choc; on a très peu de composantes, dont aucune nettement visible au-dessus du huitième partiel : la hauteur spectrale reste inférieure à 8 $\mathrm{KHz}$; la tenue du son est brève. 
Fig. 2 : Sonagramme de la valiha à cordes végétales

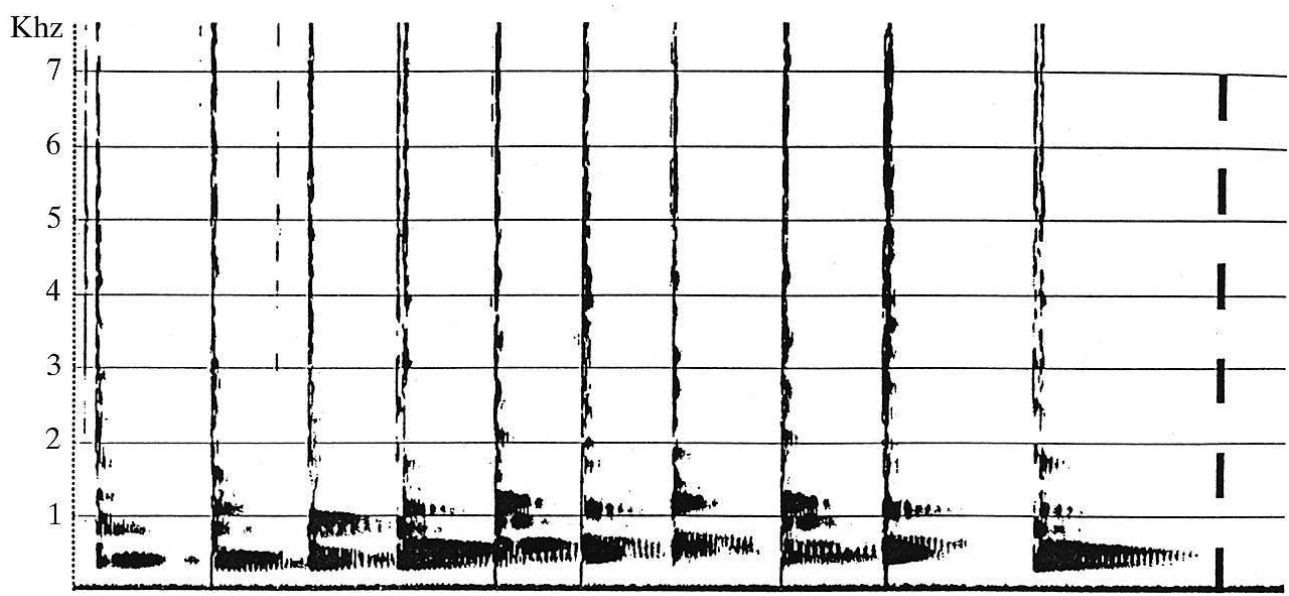

14 En revanche, l'analyse sonagraphique de la valiha à cordes métalliques (fig. 3) se caractérise par un démarrage net et simultané à l'attaque ; on peut y dénombrer plus de vingt composantes dont l'étendue va jusqu'à $16 \mathrm{Khz}$; la phase stable du son est caractérisée par une décroissance exponentielle qui dure quelques secondes.

Fig. 3 : Sonagramme de la valiha à cordes métalliques

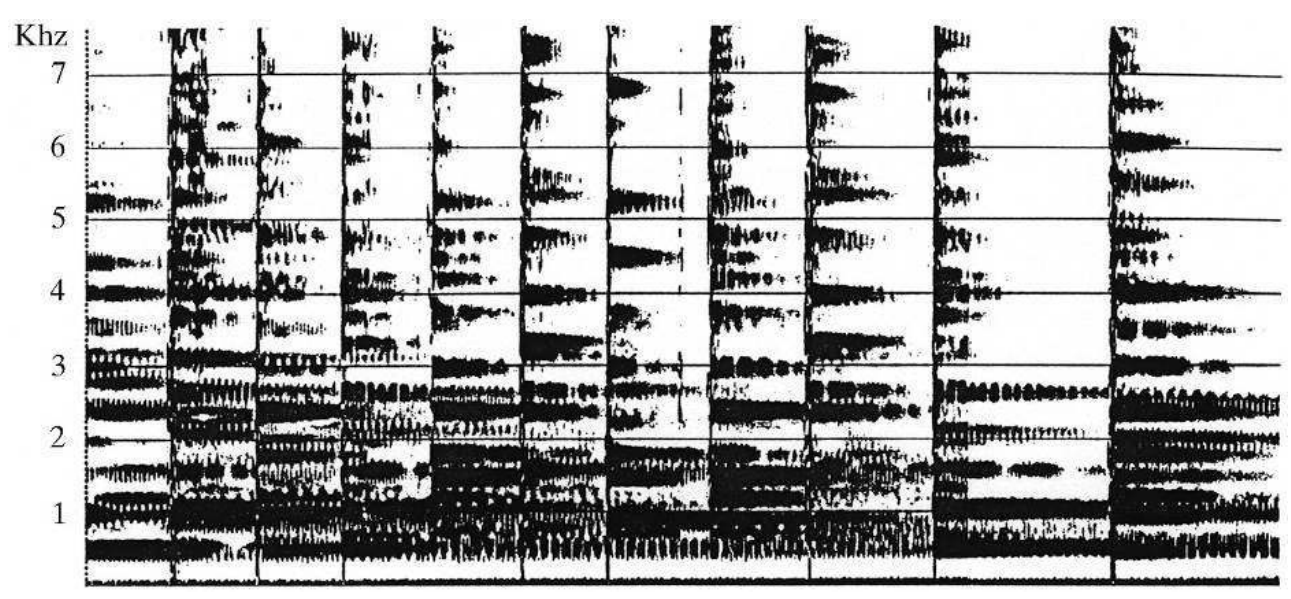

\section{La courbe du facteur de qualité de la corde}

15 Cette description sonagraphique montre la complexité et la variabilité du timbre. Pour comprendre les raisons de cette variabilité, nous avons mis ces résultats d'analyses sonagraphiques en correspondance avec des critères liés à l'analyse des matériaux. Une programmation déjà établie sur ordinateur a permis de mesurer le facteur de qualité $Q$ de chaque type de corde. Ce facteur traduit le comportement vibro-acoustique de la corde, qui est lié à des phénomènes d'amortissements internes (caractéristiques physiques du matériau) d'une part, et à des phénomènes d'amortissements externes (tel l'air ambiant) d'autre part. Le taux d'amortissement de la vibration est calculé en fonction de la fréquence $f$ et du temps de décroissance 荡. Cette méthode avait déjà été éprouvée au Laboratoire d'Acoustique Musicale sur les cordes métalliques de la tampura indienne et du clavecin (Cuesta 1990 : 1-263). Totalement novatrice sur la valiha, cette méthode a permis 
par la suite la modélisation de tous types de cordes, incluant le cas de la corde végétale (Valette et Cuesta 1993: 82-132) dont l'étude physique proprement dite n'avait pas été faite depuis Bouasse (1926).

Considérons l'effet sonore engendré par les deux types de valiha. Celle à cordes végétales donne la sensation de sons brefs, de faible intensité et de hauteurs partiellement déterminables : la sonorité est "mate et percutante». Ce constat est comparable à une analyse faite par G. Rouget sur la cithare à cordes végétales du Bénin dont l'allure sonagraphique s'apparente à celle du langage tambouriné africain (Rouget 1982: 310-323). Cela s'explique par une courbe du facteur de qualité extrêmement basse dont le maximum, situé à 170, reste relativement éloigné de la zone sensible de l'oreille (fig.4: Qth végétale $)^{2}$. En effet, le comportement vibratoire de la corde végétale induit de fortes pertes d'énergie en raison de nombreux frottements : frottements intrinsèques des fibres végétales entre elles, frottements internes dûs à la structure monobloc de l'instrument, frottements externes provoqués par la viscoélasticité de l'air. A l'opposé, la valiha à cordes métalliques laissera entendre une sonorité « claire et cristalline ». La courbe du facteur de qualité indique un maximum à 3700 situé dans la zone sensible de l'oreille (fig. 4: Qth métallique) $)^{3}$. Les caractéristiques métallurgiques de la corde, ainsi que la structure hybride de l'instrument dans laquelle la vibration est peu perturbée par le tuyau de résonance, font que l'amortissement reste relativement faible.

Fig. 4 : Comparaison entre les courbes du facteur de qualité $Q$ de la corde végétale et de la corde métallique

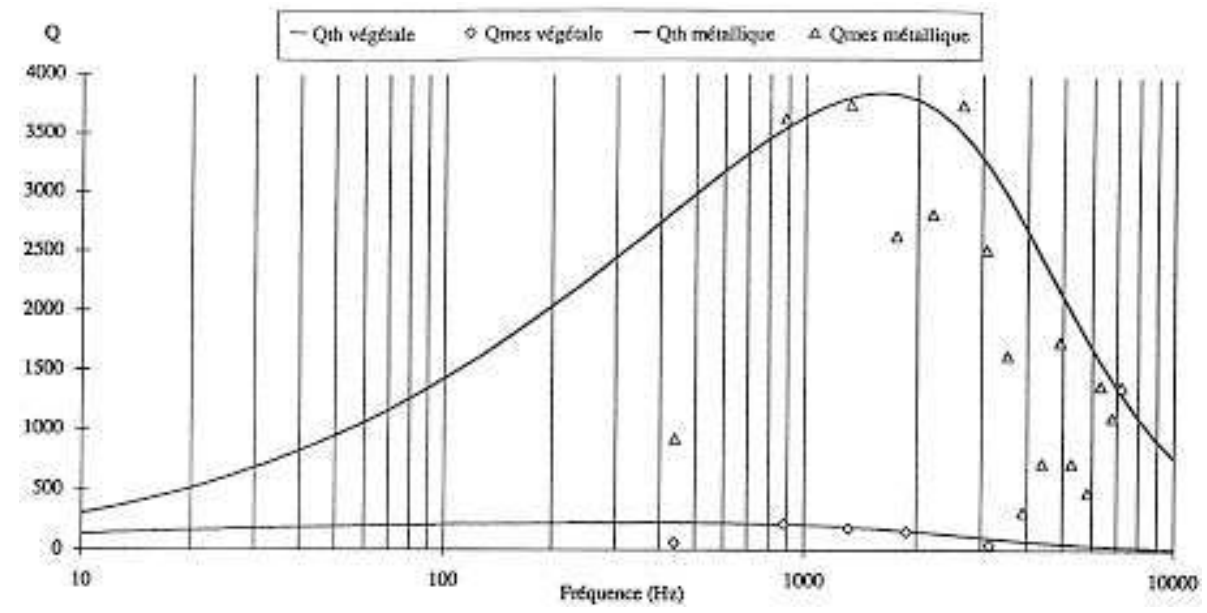

17 Ces deux types de cordes peuvent s'adapter à un usage musical, mais différent en raison d'un facteur de qualité $Q C M$ de la corde métallique 20 fois plus élévé que celui $Q C V$ de la corde végétale (Razafindrakoto $1991: 1-50$ ).

\section{Les champs de liberté des valiha}

18 Cette description du comportement vibro-acoustique de l'un et de l'autre type d'instrument nous donne les raisons pour lesquelles le timbre varie selon les matériaux de fabrication. Mais ces résultats ne nous renseignent ni sur l'utilisation musicale, ni sur la perception du timbre par les musiciens malgaches. Nous avons donc cherché à caractériser chaque paramètre constitutif du timbre à travers l'analyse des «champs de liberté » (Leipp 1965: 1-13) de ces valiha, c'est-à-dire les champs de leurs possibilités 
acoustiques et musicales. Nous introduirons parallèlement des exemples plus récents de valiha qui montrent le passage progressif d'une facture empirique à une facture de plus en plus consciente.

\section{Le champs de liberté des hauteurs}

La valiha comporte généralement seize cordes réparties sur deux octaves. Dans la région de l'Imerina, il existe plusieurs modalités d'accords dont certaines devenues obsolètes. Nous ne les évoquerons pas ici. Je retiendrai principalement l'instrument accordé en mode de do (valiha lalandava) qui demeure d'usage courant: les notes de la gamme sont alternativement réparties de part et d'autre d'une fente longitudinale taillée dans le corps de résonance et qui fait office d'ouie (fig. 5).

Fig. 5 : L'accord lalandava

20 Les relevés de fréquences ont été faits avec le stroboconn du Laboratoire. Cet appareil permet de mesurer avec précision l'écart d'une note donnée avec un étalon de référence (gamme tempérée : $l a_{3} 440 \mathrm{~Hz}$ ). On émet la note à étudier; sur l'écran de l'appareil se forme un spot lumineux circulaire qui se fractionne en un certain nombre de parties. Ce cercle tourne vers la gauche quand le son est plus grave que l'étalon et vers la droite quand il est plus aigu. On règle le spot jusqu'à ce qu'il soit immobile et on relève directement l'écart en cents, savarts ([⿷匚⿳亠口了丁3 $)^{4}$ ou commas par rapport à l'étalon (Leipp 1964 : 6).

21 Une première série de relevés fréquentiels a été effectuée avec un seul et même musicien, successivement sur la valiha à cordes végétales puis celle à cordes métalliques. La gamme réalisée sur la valiha à cordes métalliques reflète les performances de l'oreille humaine : les écarts de justesse relevés par rapport au $l a_{3} 440$ ne sont que d'environ 4 cents (ou 1䱈), valeur négligeable à l'oreille (fig. 6).

22 En revanche, les écarts de justesse observés avec des cordes végétales correctement

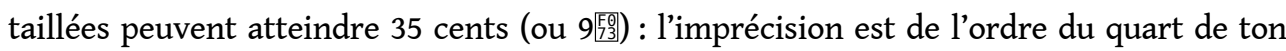
(fig. 7)!

23 Le musicien connaît de façon empirique les limites physiques de son instrument et le dégré de précision qu'il peut obtenir. Nous en avons déduit qu'indépendamment du musicien, la justesse de l'accord est liée à la nature du matériau de fabrication. La corde végétale est moins facile à accorder par rapport à la corde métallique car sa structure cellulaire produit une inharmonicité intrinsèque beaucoup plus forte que la structure homogène de l'acier. Ce qui explique pourquoi, même sur des fréquences fondamentales, la sensation d'une hauteur bien définie semble difficile à déterminer (Razafindrakoto 1997 : 272, 298). 
Fig. 6 : Relevés de fréquences sur la valiha à cordes métalliques. Les écarts observés sont peu significatifs : 4 cents, soit 1 區

\begin{tabular}{|c|c|c|c|}
\hline Notes & Valeurs minimums & Valeurs maximums & Ecarts \\
\hline do3 & 26 & 26 & 0 \\
\hline ré & 20 & 22 & 2 \\
\hline$m i$ & 13 & 13 & 0 \\
\hline$f a$ & 10 & 12 & 2 \\
\hline sol & 25 & 28 & 3 \\
\hline la & 8 & 12 & 4 \\
\hline$s i$ & 15 & 16 & 1 \\
\hline do4 & 15 & 15 & 0 \\
\hline ré & 18 & 20 & 2 \\
\hline$m i$ & 12 & 16 & 4 \\
\hline$f a$ & 8 & 10 & 2 \\
\hline sol & 20 & 20 & 0 \\
\hline la & 10 & 12 & 1 \\
\hline
\end{tabular}

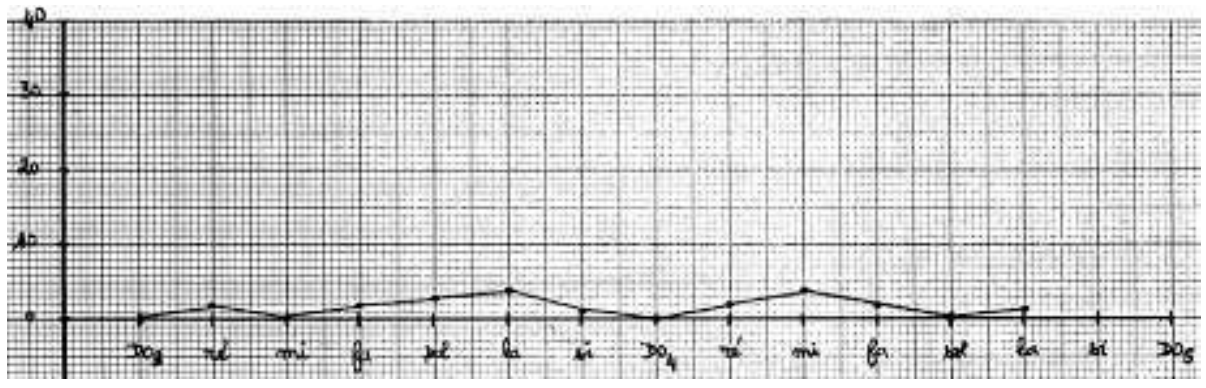

Fig. 7 : Relevés de fréquences sur la valiha à cordes végétales. Les écarts observés varient de 0 à 35 cents, soit 9 原

\begin{tabular}{|l|l|l|l|}
\hline Notes & Valeurs minimums & Valeurs maximums & Ecarts \\
\hline do3 & 10 & 20 & 10 \\
\hline ré & -3 & -3 & 0 \\
\hline \hline$m i$ & 25 & 45 & 20 \\
\hline
\end{tabular}




\begin{tabular}{|l|l|l|l|}
\hline$f a$ & -35 & -30 & 5 \\
\hline sol & -20 & -5 & 15 \\
\hline la & -25 & -10 & 15 \\
\hline si & -20 & 5 & 15 \\
\hline do4 & -10 & -3 & 7 \\
\hline ré & -30 & -20 & 20 \\
\hline$m i$ & -40 & -40 & 0 \\
\hline$f a$ & 5 & 10 & 5 \\
\hline sol & -40 & -40 & 35 \\
\hline la & -40 & 5 & 0 \\
\hline
\end{tabular}

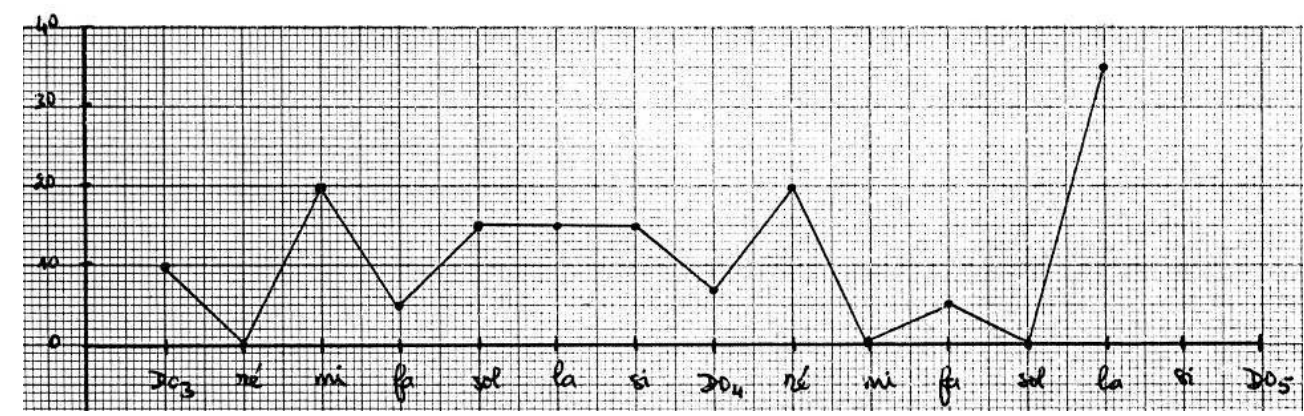

La deuxième série de relevés fréquentiels a été effectuée à partir de l'instrument à cordes métalliques seul, mais cette fois-ci avec différents musiciens enregistrés successivement. Les résultats d'analyse montrent que chaque musicien réalise un tempérament musical différent: le musicien 1 joue 20 cents plus haut, tandis que le musicien 3 joue 36 cents plus bas par rapport au la3 $440 \mathrm{~Hz}$. Le tempérament égal réalisé par le musicien 4 est une des variantes pratiquée (fig. 8). Il en résulte des couleurs différentes pour une même échelle théorique de référence (lalandava). 
Fig. 8 : Accord lalandava et tempéraments

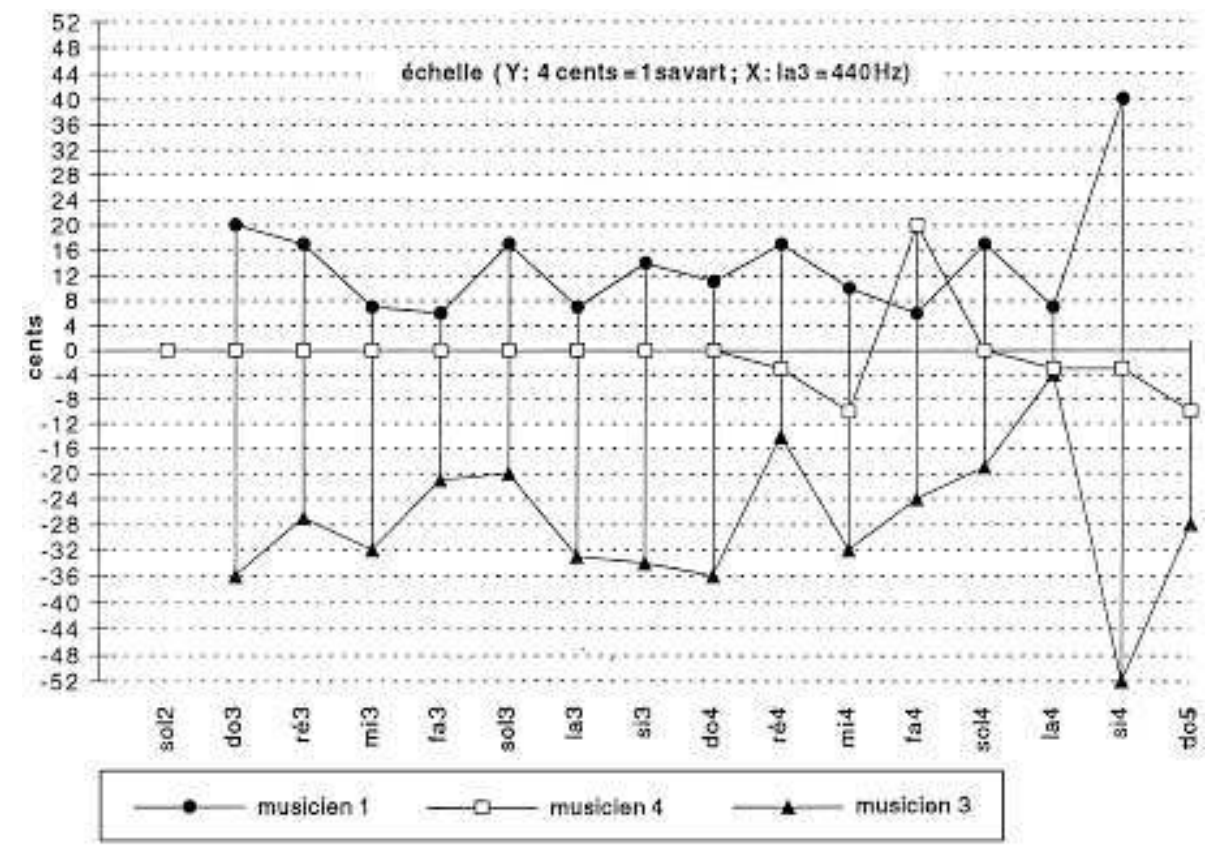

Notre analyse s'est vérifiée alors que nous enregistrions des ensembles de valiha métalliques à Antananarivo, la capitale malgache. Les musiciens qui se préparaient à jouer simultanément ne recherchaient pas l'unisson parfait. Ce principe d'accordage leur permettait de se différencier tout au long de l'exécution instrumentale: chacun d'eux était libre de réaliser son propre tempérament à partir de l'échelle lalandava. C'est ainsi que nous pouvons considérer l'existence d'un ethos chez les joueurs de valiha en Imerina pour qui l'accord traduit une forme d'expression individuelle (Razafindrakoto 1997 : 267-268).

Ce « flou » lié à la sensation de hauteur apparait comme une caractéristique pertinente de jeu du musicien. Or, il semblerait vain pour l'ethnomusicologue d'en noter toutes les variations car il n'y a pas de reproduction univoque des hauteurs : la spatialité n'est pas strictement figée pour les raisons suivantes.

D'abord, concernant la hauteur tonale : la valiha à cordes végétales introduit des écarts de justesse partiellement maîtrisables et qui peuvent sensiblement varier d'une facture à l'autre. Il est en effet particulièrement difficile de tailler une fibre végétale de façon homogène sur toute sa longueur; de plus, l'étude vibro-acoustique de l'instrument montre que des frottements intrinsèques à la nature du matériau agissent inévitablement sur la justesse de l'accord (voir l'analyse précédente du facteur de qualité Q). Ensuite, il n'y a pas de tempérament fixe : la finesse d'accordage que l'on peut obtenir sur une corde métallique parfaitement homogène comme l'acier montre que seul entre en ligne de compte le jugement individuel du musicien. L'accord introduit un ethos des tempéraments propre à chaque musicien. Enfin, concernant la hauteur spectrale : un modèle de facture particulièrement originale a retenu mon attention. Il s'agit d'une valiha dont chaque note est composée d'un chœur de deux ou trois cordes métalliques légèrement désaccordées entre elles (dispositif semblable au luth de la Renaissance ou encore aux notes aiguës du piano). L'attaque simultanée de chaque doublé ou triplé de cordes engendre une sensation de rugosité peu usuelle avec une corde métallique. Ici, le musicien révèle son 
intention de brouiller toute sensation de hauteur pure en recherchant un timbre à l'effet "granuleux ».

Nous pouvons en conclure que la justesse, liée au degré d'inharmonicité des cordes de la valiha, influe sur la sensation de timbre. Un procédé d'analyse numérique de la corde vibrante a été expérimenté à ce sujet sur la valiha avec la mise au point d'un programme informatique approprié MATLAB. Ce programme permet d'avoir une plus grande étendue spectrale et une meilleure précision sur le degré d'inharmonicité (Oudot 1995 : 1-48).

\section{Le champ de liberté des durées}

L'évolution temporelle des sons de la valiha à cordes végétales et celle de la valiha à cordes métalliques sont très différentes. A la lecture des sonagrammes, nous avons vu que ce temps est extrêmement bref sur la corde végétale. Cet amortissement rapide est dû à la structure-même du matériau (rappelons-nous les frottements auxquels il est soumis) en dépit d'une énergie vibratoire importante délivrée à l'attaque(cf. fig. 2).A l'opposé, la vibration de la corde métallique affiche une décroissance exponentielle. Compte-tenu des caractéristiques métallurgiques de l'acier et des pertes d'énergie moins importantes, l'amortissement se fait progressivement, les composantes aiguës décroissant plus rapidement que les composantes graves (cf. fig. 3). Cette caractéristique est exploitée sur le plan musical par une maîtrise de la durée des sons de la corde métallique : laisser vibrer cette dernière ou étouffer sa vibration avec la pulpe du doigt. Cette technique de jeu pratiquement inusitée sur la valiha à cordes végétales permet de contraster sons tenus et staccato, de prolonger les basses (fig. 9), de créer des effets de trémolo (fig. 10) (Razafindrakoto 1997 : 232-233).

Fig. 9 : Jeu lié et piqué sur la valiha à cordes métalliques

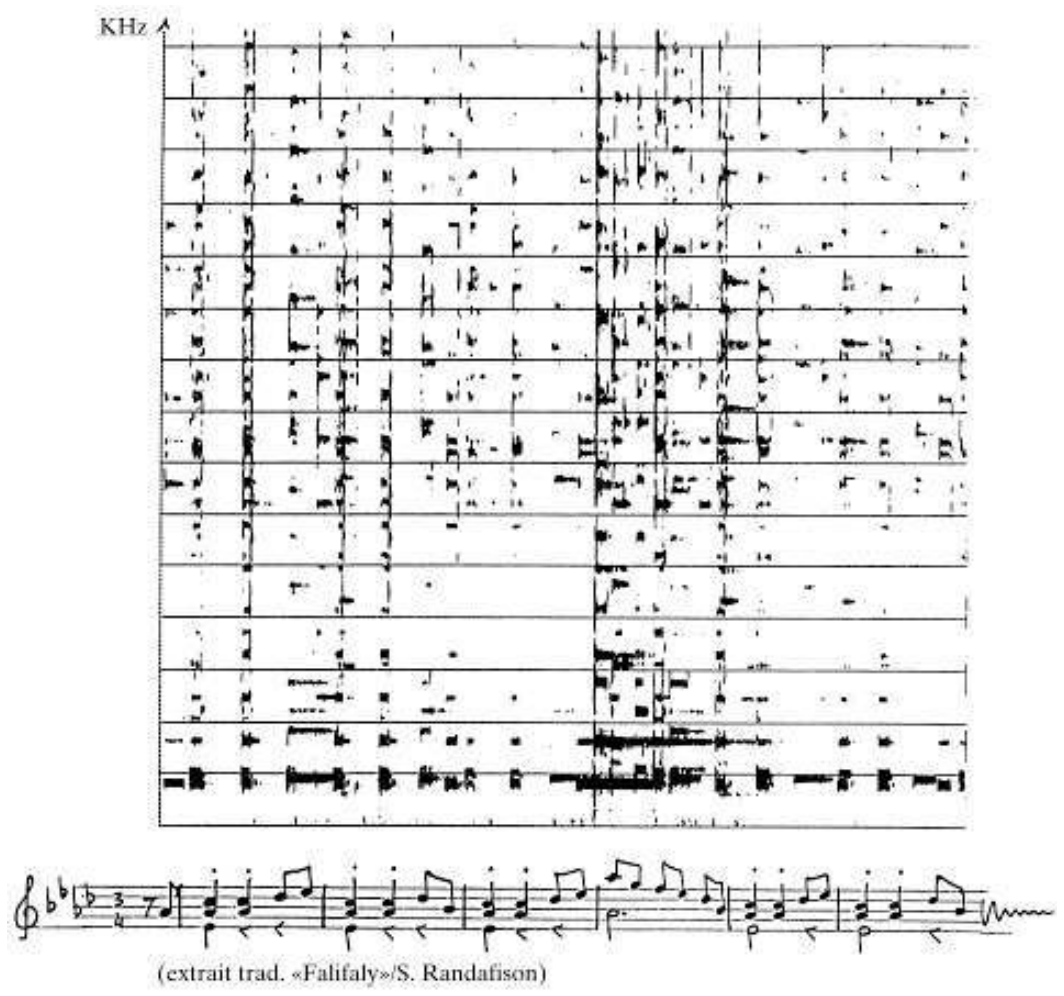


Fig. 10 : Trémolo sur la valiha à cordes métalliques

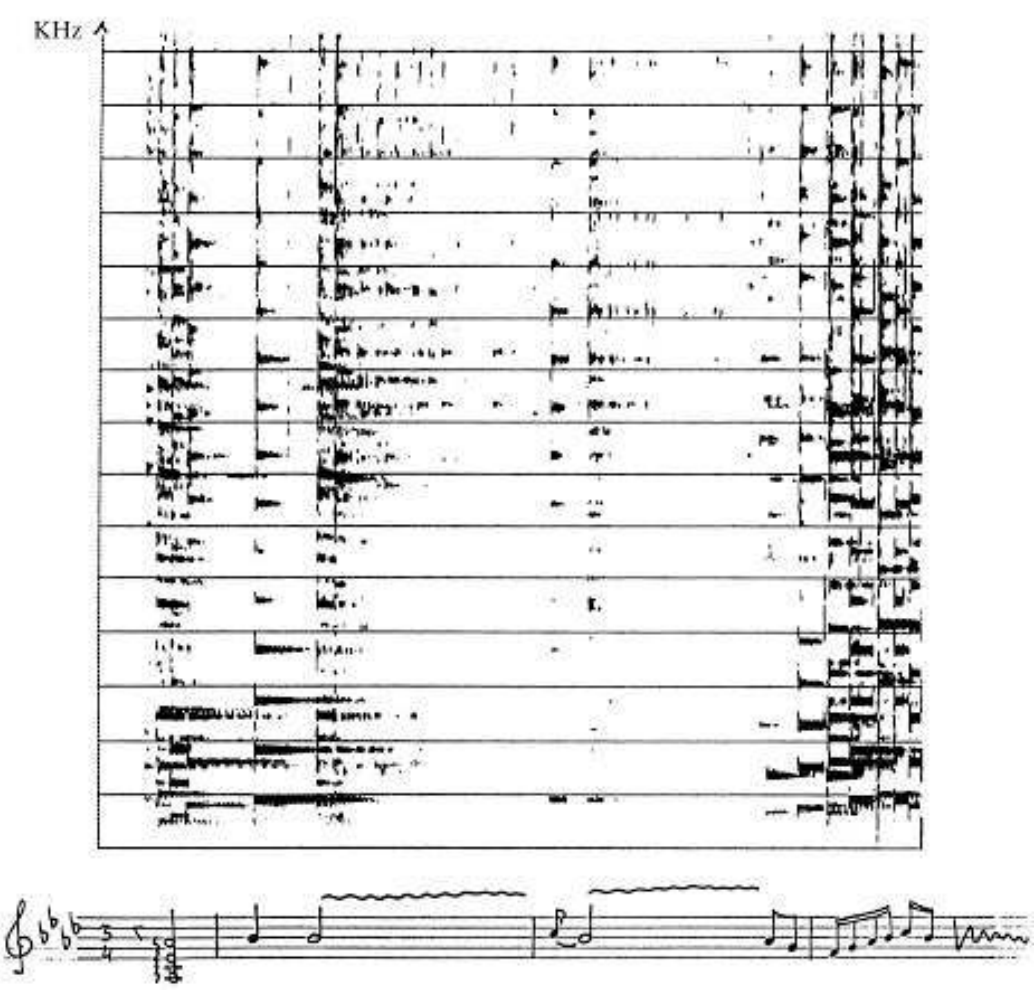

Deux variantes de facture extrêmement intéressantes montrent que l'évolution temporelle est un paramètre pertinent du point de vue des musiciens. La première est un modèle de valiha dont les cordes métalliques sont montées sur un tuyau non pas en bambou comme le veut l'usage, mais sur un corps de résonance en tôle (de forme tubulaire dans la région de l'Imerina, parallélépipédique dans d'autres régions de l'île). La grande homogénéité de ce couplage métal/métal favorise des tenues de sons encore plus longues, intéressantes pour les bourdons.

31 Le second modèle consiste à contrarier la vibration naturelle des cordes métalliques en les enserrant dans un bracelet en caoutchouc (valiha élastique). Cet "étouffement " volontaire des cordes métalliques permet d'obtenir une allure sonagraphique étonnemment similaire à celle de la valiha à cordes végétales : recréation judicieuse du comportement vibratoire et de la sonorité de l'instrument originel avec des matériaux modernes. La seule différence est l'émergence de partiels aigus correspondant à la position de l'élastique sur les cordes (Razafindrakoto $1997: 226-229$ ).

\section{Le champ de liberté des intensités}

L'amplitude, c'est-à-dire l'écart entre l'intensité la plus faible et la plus élevée, peut être représentée graphiquement par une courbe qui décrit l'évolution du niveau sonore dans le temps. Les choix de lutherie interviennent dans cette caractéristique. Ainsi, l'amplitude varie selon le type de couplage "corps de résonance/cordes". Par exemple, dans le couplage « bambou/bambou » qui introduit des pertes d'énergie importantes, l'amplitude reste relativement faible. 

le musicien obtiendra des dynamiques de jeu beaucoup plus contrastées, visibles par la densité des composantes spectrales sur les sonagrammes (fig. 11).

Fig. 11 : Opposition d'intensités dans le jeu de la valiha à cordes métalliques

Cette qualité sonore sera musicalement exploitée en variant les techniques d'attaque des cordes. Le musicien obtiendra une intensité douce avec la pulpe du doigt, plus forte avec l'ongle. De même, l'attaque à différents endroits de la corde (bas, milieu, haut) modifie le contenu spectral d'un même son et favorise des zones formantiques (Razafindrakoto 1997 : 286-288).

Malgré les variantes d'intensité offertes par ces différents couplages, un problème technique particulier tel que le dosage de l'intensité sonore lié à une nouvelle spatialisation de la musique se pose aux instrumentistes: distance des instrumentistes entre eux, et des instrumentistes par rapport à l'auditoire. Pour répondre aux exigences du monde moderne, la valiha est couramment électrifiée en situation de concert : l'enjeu consiste à conserver le meilleur équilibre entre un instrument d'intimité et de nouveaux espaces acoustiques. Une comparaison avec des expériences de sonorisation de la guitare faites au Laboratoire montre que l'on peut obtenir des solutions optimales en fixant le micro-contact sur la «tête » ou sur le «pied » de la valiha : sur ces zones les plus rigides du tuyau de résonance, le son garde le maximum de sa richesse harmonique. Dans la pratique, ce sont effectivement les endroits privilégiés par les musiciens (Razafindrakoto 1997 : 295-296). 


\section{Le timbre, une donnée complexe et modulable} cordes végétales et celle à cordes métalliques. Les analyses faites avec différents outils (sonagraphe numérique, accordeur électronique, programmations à l'ordinateur) offrent une description physique et acoustique fine et précise de chaque paramètre sonore. La pertinence de cette démarche a été maîtrisée sur deux plans. D'abord, en veillant à ce que les valeurs retenues restent significatives à l'oreille. Ensuite, en cherchant ce qui, d'un point de vue qualitatif, motivait le choix des artisans-musiciens. manière de faire moduler le son fixe, non entretenu, des cordes pincées de la valiha. La variation d'un seul des trois paramètres constitutifs du timbre, menée à travers l'analyse des champs de liberté de la valiha, suffit à le modifier. C'est un phénomène dont les musiciens ont parfaitement conscience. Si bien que cette connaissance influe sur leur manière de façonner les instruments pour obtenir tel ou tel effet sonore. J'ai récapitulé l'exploration du timbre dans le tableau ci-contre, en mettant en évidence l'exploitation systématique des différentes composantes.

variantes de facture révèlent que le timbre instrumental n'est pas seulement considéré comme une valeur ajoutée aux paramètres musicaux usuels de hauteur, de durée et d'intensité. Le timbre est considéré par ces artisans-musiciens malgaches comme une donnée structurante de la musique en raison de sa complexité (il contient tous les paramètres sonores) et de sa malléabilité (la modification d'une seule de ses composantes suffit à le faire varier).

Les musiciens ont trouvé dans la valiha à cordes métalliques un compromis satisfaisant sur le plan de la facture et sur les qualités de jeu. En effet, l'intérêt que présente la corde métallique (prélevée sur un câble de frein de vélo!) tient en premier lieu à ses caractéristiques technologiques: ce matériau est apprécié pour sa résistance et sa stabilité hygrométrique. Sur le plan musical, la corde métallique offre une meilleure perception des rapports de hauteurs (grave/aigu), de durées (longues/brèves) et d'intensités (fort/doux) permettant de mettre en valeur des effets de jeu qui demeurent peu perceptibles sur la corde végétale (oppositions de registres, de durées et d'intensités). Ce qui fait d'elle un matériau unanimement adopté par les musiciens malgaches.

\begin{tabular}{|l|ll|l|}
\hline $\begin{array}{l}\text { Composantes } \\
\text { sonores/ } \\
\text { Principe de } \\
\text { facture }\end{array}$ & $\begin{array}{l}\text { Extrapolation des possibilités } \\
\text { naturelles de la corde métallique }\end{array}$ & $\begin{array}{l}\text { Procédés artificiels appliqués } \\
\text { à la corde métallique : }\end{array}$ \\
\hline $\begin{array}{l}\text { Evolution } \\
\text { spectrale }\end{array}$ & $\begin{array}{l}\text { Valiha «à cordes filées " } \\
\text { (enrichissement des composantes } \\
\text { harmoniques des sons graves de } \\
\text { l'instrument) }\end{array}$ & $\begin{array}{l}\text { Valiha «élastique » (appauvrissement de } \\
\text { la hauteur spectrale des cordes } \\
\text { cahoutchouc) }\end{array}$ \\
\hline
\end{tabular}




\begin{tabular}{|l|l|l|l|}
\hline $\begin{array}{l}\text { Evolution } \\
\text { temporelle }\end{array}$ & $\begin{array}{l}\text { Valiha «tôle (allongement du } \\
\text { temps de vibration des cordes } \\
\text { métalliques avec un corps } \\
\text { de résonance en tôle) }\end{array}$ & $\begin{array}{l}\text { Valiha «à cordes sympathiques » } \\
\text { (inharmonicité accrue par les doublés et } \\
\text { triplés de cordes métalliques) }\end{array}$ \\
\hline $\begin{array}{l}\text { Evolution } \\
\text { dynamique }\end{array}$ & $\begin{array}{l}\text { Valiha «tôle (renforcement } \\
\text { naturel des composantes spectrales } \\
\text { grâce au couplage des cordes } \\
\text { métalliques avec un corps de } \\
\text { résonance en tôle) } \\
\text { Valiha «électrifiée (adaptation de } \\
\text { l'intensité via un micro-contact fixé } \\
\text { sur l'instrument) }\end{array}$ & $\begin{array}{l}\text { Valiha «élastique » (étouffement de la } \\
\text { bracelet en caoutchouc) }\end{array}$ \\
\hline $\begin{array}{l}\text { Résultats } \\
\text { sonores }\end{array}$ & $\begin{array}{l}\text { S'opposent au timbre de la valiha à } \\
\text { cordes végétales adjonction } \\
\text { d'accessoires spécifiques }\end{array}$ & $\begin{array}{l}\text { Evoquent le timbre de la valiha à cordes } \\
\text { végétale }\end{array}$ \\
\hline
\end{tabular}

\section{La fonction esthétique des timbres de la valiha}

$\mathrm{Au}$ terme de cette étude acoustique, il convient d'aborder la question du timbre de la valiha dans sa signification fondamentale. Pour les artisans-musiciens merina, le souci du timbre est une préoccupation majeure, que l'ensemble des transformations liées à la facture de l'instrument ne rend pas explicite de prime abord. Or, le timbre devient un élément constitutif du répertoire de la valiha dès lors qu'ils lui confèrent une fonction et une signification musicale précises. Quels sont donc les traits culturellement pertinents pour les artisans-musiciens?

\section{Le timbre comme élément de variation et de stylisation}

41 L'utilisation quasi-généralisée de la corde métallique (d'usage courant depuis le début du $\mathrm{XX}^{e}$ siècle) masque une réalité sous-jacente qu'une étude complète (organologique, acoustique et socio-historique) de l'instrument a permis de mettre à jour : les variantes de facture sont conçues par rapport à la valiha originelle, à cordes végétales. Il en résulte des sonorités façonnées soit en opposition (colonne de gauche), soit en évocation (colonne de droite) au timbre de cet instrument de référence (Razafindrakoto 1997 : 299-301) comme on peut le retrouver dans le tableau synoptique précédent - bien que je n'aie sélectionné ici que quelques cas concrets de facture instrumentale.

La responsabilité de l'artisan-musicien est de taille car il utilise " une palette sonore » définie par la facture de son instrument et par ses techniques de jeu. Les différents qualificatifs juxtaposés au mot "valiha» révèlent une conscience aiguë de l'artisanmusicien qui, par le biais de la facture instrumentale, exploite de façon systématique les champs de liberté de l'instrument. Cette expérience empirique, fondée sur un savoir-faire ancestral, montre ici que le timbre est perçu comme une donnée complexe et modulable où la référence au timbre de la valiha originelle à cordes végétales demeure sous-jacente et délimite le cadre des variantes sonores de l'instrument. 
terrain m'amène à constater deux faits : premièrement, la valiha est souvent remplacée par des instruments d'importation occidentale tels que l'accordéon diatonique (une observation semblable a été faite par Koechlin sur les populations du Sud de Madagascar : 1967-69) dans la plupart des cérémonies rituelles. Deuxièmement, il est désormais courant de retrouver la valiha, instrument séculaire, dans des zones urbanisées. Dans le premier cas, la population s'accommode d'instruments dont elle dispose sur le moment: un instrument peut alors se substituer à un autre. Dans le deuxième cas, l'instrument est présent dans des lieux où la musique circule de façon plus diversifiée. Mais le constat est le même : la musique traditionnelle demeure vivante et continue à être jouée.

approche de la valiha révèle une conscience profonde du son dans la population merina : le timbre est vécu « de l'intérieur », dans sa nature première c'est-à-dire comme source de la musique. A travers cette matière sonore, les musiciens cherchent, au cours de l'histoire, à faire corps avec les réalités mouvantes de l'environnement. C'est parce qu'elle renvoie à la mémoire collective, c'est-à-dire à un vécu, que la sonorité de la valiha acquiert toute sa puissance. C'est ainsi que le timbre de la valiha est adapté à une réalité socioculturelle.

L'étude de ce répertoire nécessite donc d'admettre la possibilité d'une évolution de linstrument de musique traditionnel et des musiques qui lui sont associées : ils ne sont renouvellement de sa facture. L'instrument véhicule un répertoire ancien en même temps soit jouée sur différents types de valiha. Un même thème musical peut être "traité » différemment selon la valiha utilisée : tenues de sons plus ou moins longues sur le plan rythmique, opposition ou complémentarité de registres sur le plan mélodique, création de plans sonores par des variations d'intensités sur le plan dynamique, etc.

de sons que l'instrument peut générer et qui conditionnent la stylisation des pièces musicales. Le traitement du timbre que l'on retrouve dans d'autres musiques extra129-130), est une donnée à prendre en compte dans l'analyse et la notation d'un répertoire. Il détermine la manière de façonner mélodies, rythmes et dynamiques, ainsi que celle de faire varier le jeu d'un même répertoire rendu ainsi différent en apparence.

\section{Le pouvoir expressif du timbre}

culturellement significatif pour les artisans-musiciens malgaches. Ce «culturellement » répertoire musical proprement dit. En amont, nous avons vu l'importance accordée à la source sonore. En aval, dans la mesure où l'instrument est relié au système de pensée et au contexte ethnique qui l'a fait naître (Schaeffner 1980), l'ethnomusicologue doit se soucier de la fonction de l'instrument au sein de la culture étudiée.

En résumant très sommairement, le timbre de la valiha à cordes végétales se réfère au monde des Anciens : dans la mémoire de la population merina, la valiha évoque les rites ancestraux et les valeurs communautaires qui y sont associées. Au contraire, le timbre de 
la valiha à cordes métalliques (conçu entre le milieu du $\mathrm{XIX}^{\mathrm{e}}$ et le début $\mathrm{du} \mathrm{XX}^{\mathrm{e}}$ siècle) marque une nouvelle sensibilité à la modernité. La période contemporaine a engendré des variantes avec le timbre de la valiha « élastique » qui simule un retour aux sources et celui de la valiha dite "à cordes sympathiques" qui symbolise les aléas du monde actuel (modèles de facture apparus en Imerina dans les années 1980-90).

De nombreuses pièces musicales issues du cadre rituel concourent toujours à véhiculer les valeurs communautaires; les procédés d'organisation interne des éléments musicaux (composition) restent stables mais les styles, qui renvoient à une valeur expressive de la musique, se diversifient selon les époques et les personnalités musicales. Il y a une actualisation permanente où timbres et techniques particulières de jeu constituent les variantes essentielles du répertoire. Le timbre de la valiha a ainsi évolué en fonction de différentes époques, en veillant à ce que « le musical, [soit] le sonore construit et reconnu par une culture » (Molino 1975: 53). L'artisan-musicien doit assurer cette continuité historique, c'est-à-dire maintenir le statut de l'instrument en trouvant des solutions de compromis techniques et esthétiques par rapport à un paysage socioculturel en constante évolution (Razafindrakoto 1997 : 388-390).

Cette recherche montre la dialectique qui existe entre le pouvoir structurel du son considéré pour lui-même d'une part, et le pouvoir expressif du son rattaché aux conditions extérieures au musical d'autre part. Le timbre est ici révélé comme une donnée essentielle dans l'évolution diachronique de la valiha en Imerina et dans le traitement stylistique du répertoire traditionnel. Il est conçu comme un élément à la fois structurel et émotionnel de premier ordre qui va s'enraciner dans la conscience et la mémoire collectives. Cette recherche liée à l'efficacité du contenu expressif du son rejoint cette citation de Lutoslawski : «la seule chose qui soit importante et légitime dans une œuvre musicale, c'est ce qui peut aider à produire un résultat précis au moment de la perception » (Bayer 1987 : 195).

\section{En conclusion}

Le timbre instrumental est une donnée musicale essentielle que les outils technologiques actuels permettent de décrire avec finesse et précision. Les correspondances qui peuvent être généralement établies entre la représentation sonagraphique et la notation solfégique traditionnelle fournissent des éléments d'information utiles sur l'aspect quantitatif du son. Mais l'aspect qualitatif représente encore une source de difficultés pour une transcription complète. La seule indication de l'instrument paraît insuffisante lorsque le timbre est, dans le cas de la valiha, traité comme élément fondamental de variations et de stylisation du répertoire. Une solution possible consisterait à caractériser la «palette sonore» du musicien après que l'ethnomusicologue l'ait préalablement déterminée, en prenant en compte certains points déterminants dans la production du son. Le choix des matériaux de fabrication (bambou, acier, tôle...), des dispositifs particuliers de jeu (élastique, doublés ou triplés de cordes, micro-contact...) doivent être spécifiés. De même, les modes de jeu doivent être précisés en notant les types d'attaque correspondant à tel geste instrumental (pincement avec la pulpe du doigt, avec l'ongle, avec ou sans étouffement de la corde, râclement des cordes...). 


\section{BIBLIOGRAPHIE}

BAYER Francis, 1987, De Schönberg à Cage. Paris : Klincksieck.

BOUASSE M., 1926, Cordes et membranes. Paris : Delagrave.

CASTELLENGO Michèle, 1994, «Perception du signal musical ». Bulletin de l'Union des Physiciens (Paris) $761:$ 197-209.

CHION Michel, 1983, Guide des objets sonores : Pierre Schaeffer et la recherche musicale. Paris : BuchetChastel ; INA/GRM.

CUESTA Christian, 1990, Corde vibrante isolée mécaniquement, amortissement, non-linéarités ; application au clavecin et à la tampura. Thèse d'Acoustique de l'Université du Maine, 263.

DEHOUX Vincent, 1987, «L'un dans l'autre : improvisation et répétition dans un répertoire instrumental centrafricain ", in L'improvisation dans les musiques de tradition orale. Paris: Selaf, 123-132.

DOMENICHINI Michel, 1984, " Valiha », in Stanley Sadie ed.: New Grove Dictionary of Musical Instruments. London : Macmillan, vol. 3 : 705-706.

LEIPP Emile, 1964, " Appareillages et méthodes en acoustique musicale ». Bulletin du GAM (Paris) 4, 9., 1965, « Les champs de liberté des instruments de musique ». Bulletin du GAM (Paris) 10, 13.

MOLINO Jean, 1975, « Fait musical et sémiologie de la musique », Musique en jeu (Paris) 17 : 37-62.

OUDOT Marine, 1995, Mesure d'impédance par comparaison d'inharmonicité (application à la valiha). Mémoire de DEA/ATIAM (Acoustique, Traitement du signal et Informatique Appliqués à la Musique), 48.

RAZAFINDRAKOTO Jobonina, 1991, Comparaisons entre les timbres de la valiha à cordes végétales et de la valiha à cordes métalliques : cithares tubulaires de Madagascar. Mémoire d'Acoustique Musicale du Conservatoire National Supérieur de Musique de Paris, 50.

RAZAFINDRAKOTO Jobonina, 1997, La valiha de Madagascar : tradition et modernité en Imerina de 1820 à 1995 (Etudes organologique, acoustique et socio-historique). Thèse de doctorat de l'Université Paris IV-Sorbonne, 463.

ROUGET Gilbert, 1982, "Cithares et glissando. Nouvelles données sur le chromatisme au Bénin », Les fantaisies du voyageur. Paris : Société Française de Musique : 310-323.

SACHS Curt, 1938, Les instruments de musique de Madagascar. Paris : Institut d'Ethnologie.

SCHAEFFER Pierre, 1966, Traité des objets musicaux. Paris : Seuil.

SCHAEFFNER André, 1980, Origine des instruments de musique. Paris, La Haye, New-York : Mouton (2 e éd.).

VALETTE Claude, CUESTA Christian, 1993, Mécanique de la corde vibrante. Paris : Hermès.

\section{Références discographiques}

DOMENICHINI Michel, 1985, Marovany/Valiha : Madagascar. France : GREM G7507, CD et livret. 
DUVELLE Charles, 1963, Valiha-Madagascar. Paris : OCORA 18, disque vinyl et livret.

KOECHLIN, 1967-69, Possession et poésie à Madagascar. Paris : OCORA 83, disque vinyl et livret ; rééd.1982 en CD.

\section{NOTES}

1. Conférence de clôture de l'année 1998-1999, consacrée à la Valiha de Madagascar. Tananarive : Académie Malgache, juillet 1999.

2. Les mesures que nous avons faites sont indiquées par les points $Q_{\text {mes. végétale }}$; la courbe théorique correspondante par $Q_{\text {th végétale }}$.

3. Les mesures sont indiquées par les points $Q_{\text {mes.métallique }}$; la courbe théorique correspondante par $Q_{\text {th métallique }}$.

4. Le cent est utile pour la précision des mesures, tandis que le savart (s) est une unité plus pratique car il correspond à une réalité perceptive (sensation d'intervalle) : 1 octave $=1200$ cents $=300 \mathrm{~s} ; 4$ cents $=1 \mathrm{~s}$.

\section{RÉSUMÉS}

La valiha, cithare tubulaire de Madagascar, est un instrument de musique séculaire largement répandu dans l'île. Sa pratique est particulièrement vivace en Imerina, région des Hautes terres centrales : en témoigne la richesse de la terminologie locale qui sert à désigner des variantes de factures et de jeu.

Le timbre est révélé comme une donnée structurellement et esthétiquement pertinente dans le jeu de la valiha en Imerina. Or, tout renouvellement du timbre a des répercussions plus ou moins profondes sur le plan stylistique. L'influence réciproque qui existe entre les procédés de facture et les caractéristiques de jeu a été mise en évidence par une analyse des « champs de liberté » de la valiha. Le recours à des outils et des méthodes spécifiques à l'acoustique musicale a permis de décrire ces phénomènes, puis de les confronter à la pratique musicale des artisans-musiciens, afin d'en dégager la pertinence. La complexité et la malléabilité du timbre instrumental ont permis aux artisans-musiciens de le façonner au cours de l'histoire, réactualisant ainsi le répertoire par rapport à un contexte socio-culturel en constante évolution. La référence au timbre de la valiha originelle à cordes végétales reste sous-jacente et guide les artisans-musiciens dans leurs choix de facture et de jeu : certaines variantes sont conçues en opposition, d'autres en évocation à cet instrument de référence.

Spécifier l'instrument comme il est d'usage dans les partitions d'orchestre classiques paraît insuffisant et la difficulté que pose la notation des variantes stylistiques liées au timbre instrumental n'est pas entièrement résolue. 


\section{AUTEUR}

\section{JOBONINA RAZAFINDRAKOTO}

Jobonina Razafindrakoto, 38 ans, a suivi ses études de musicologie à l'Université Paris IV/ Sorbonne. Elle approfondit ses recherches sur la cithare tubulaire de Madagascar (valiha) dans la classe d'acoustique musicale du Conservatoire National Supérieur de Musique de Paris. Elle est titulaire d'un doctorat de musicologie. Le sujet de sa thèse porte sur l'adaptation des artisansmusiciens malgaches à la modernité, à travers l'évolution de la valiha en Imerina (Hautes Terres centrales de Madagascar). Ce projet a bénéficié d'une bourse du Ministère de la Culture et l'encadrement technique du Laboratoire d'Acoustique Musicale (UMR 9945 : CNRS/Université Paris VI/Ministère de la Culture). Elle a favorisé la publication d'un ouvrage sur la valiha

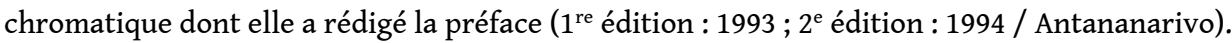

